\title{
Multi-objective Optimization of Product Life-Cycle Costs and Environmental Impacts
}

\author{
Daniele Cerri ${ }^{1}$, Marco Taisch ${ }^{1}$, and Sergio Terzi ${ }^{2}$ \\ ${ }^{1}$ Politecnico di Milano, Department of Management, Economics and Industrial Engineering \\ Piazza Leonardo da Vinci 32, \\ 20133, Milano, Italy \\ ${ }^{2}$ Università degli Studi di Bergamo, Department of Industrial Engineering \\ Viale Marconi 5, \\ 24044, Dalmine (Bergamo), Italy \\ \{daniele.cerri, marco.taisch\}@polimi.it, \\ sergio.terzi@unibg.it
}

\begin{abstract}
The present paper discusses the development of a model to multiobjective optimization of product life-cycle costs and environmental impacts. In modern world European companies need new competitive factors. Costs along the life-cycle and sustainability could provide two of these. A model to optimize costs and environmental impacts could play a relevant role for engineering the life-cycle of a product. Within this context, the paper conducts a state of the art review of existing solutions. In the end a model is proposed.
\end{abstract}

Keywords: LCA, Life Cycle Assessment, LCC, Life Cycle Costing, Multi Optimization, Genetic Algorithms, Product Life Cycle.

\section{Introduction}

Today, in the modern global world, European companies need to find new competitive factors for facing the low-cost pressure of emerging countries. Sustainability, more and more pushed by international regulations (e.g. Kyoto Protocols, European Directives, etc.), could provide one of these factors: being able to develop eco-friendly, energy-efficient and green products before the others could give a competitive advantage to European industries for the next years. In this research, companies can be supported by methodologies already well-known in literature, like Life Cycle Costing (LCC) and Life Cycle Assessment (LCA), which permit to perform cost and environmental analysis for developing more sustainable products. However, most of the researches available in literature are not able to guarantee the reaching of the optimal solution, but in most of the experiences LCC and LCA are just used for simple evaluations. This paper aims to fill this gap, proposing a model that optimizes product life-cycle costs and environmental impacts at the same time. The paper is organized as follows: Section 2 illustrates the current state of the art of LCC, LCA and optimization methods; Section 3 describes the proposed model, with real application; finally, Section 4 concludes the paper. 


\section{State of the Art}

Life Cycle Cost and Life Cycle Assessment are well-known methodologies in the relevant literature. Both have been developed since the "60ies: LCC are "cradle-tograve" costs summarized as an economic model of evaluating alternatives for equipment and projects [3]; LCA is a technique to assess environmental impacts associated with all the stages of a product's life from-cradle-to-grave [13]. For this paper, it is interesting to analyze the state of the art of optimization applied to LCC and LCA. 39 papers for LCC and 40 papers for LCA, from the last 15 years, have been analyzed. We have classified the contributions in three clusters: (i) simple application of the methodology, (ii) use of a software, (iii) optimization. The first cluster considers papers that barely apply the methodology (LCC or LCA). The second cluster includes contributions that use a software to calculate costs and / or environmental impacts. The third cluster takes into account papers that optimize product life-cycle's costs and / or environmental impacts.

We observe that only few papers consider optimization issues. In percentage, only $20.51 \%$ of LCC literature treats about optimization, reduced to $10 \%$ in LCA. Evidently, this research area is still emerging and this paper can give a contribution.

Another interesting information is the massive use of software in LCA, while in LCC is practically missing. Use of software in LCA is justified by the increased complexity of the methodology, compared to LCC. The most popular LCA software are: SimaPro, GaBi Software and LCAiT.

Focusing on papers dealing with optimization, the used optimization methods are: Linear Programming, Genetic Algorithm and Particle Swarm Optimization.

In [1] Azapagic and Clift applied 2 algorithms: a single objective linear programming model to optimize LCA and a multi-objective linear programming model to optimize LCA and profit of a chemical plant that produces thermoplastic materials. In [2] the previous authors developed a multi-objective linear programming to optimize LCA (understood as Global Warming Potential), costs and total production of a chemical system that produces 5 boron products. Cattaneo [4] instead use a single objective linear programming model to optimize LCC of a train traction system.

Other 6 papers use genetic algorithm instead of linear programming.

Gitzel and Herbort [8] applied a genetic algorithm to optimize LCC of a DCS (Distributed Control System), using different GA variants. Hinow and Mevissen [9] use genetic algorithm to optimize LCC of a substation, improving the maintenance activities. In Kaveh et al. [10] genetic algorithms, exactly NSGA-2, is used to perform a multi-objective optimization of LCC and initial costs of large steel structures. Here it is possible to see the strong trade-off between initial costs and life cycle costs. Frangopol and Liu [7] and Okasha and Frangopol [12] applied a multi-objective genetic algorithm to optimize three different objectives: LCC, lifetime condition index value and lifetime safety index value for [7]; LCC, minimum redundancy index and maximum probability of failure for [12]. They are applied on structural maintenance. Dufo-Lopez et al. [6] instead applied the Strength Pareto Evolutionary Algorithm (SPEA) to the multi-objective optimization of a stand- 
alone PV-wind-diesel system with batteries storage. The objectives to be minimized are the levelized cost of energy (LCOE) and the equivalent $\mathrm{CO}_{2}$ life cycle emissions (LCE). LCE can be viewed as LCA.

Finally, two papers use the particle swarm optimization.

Kornekalis [11] use a multi-objective particle swarm optimization to the optimal design of photovoltaic grid-connected systems (PVGCSs), maximizing Net Present Value (NPV) and the pollutants gas emissions avoided due to use PVGCSs (this can be compared to LCA); Wang et al. [15], instead, applied a Particle Swarm Optimization to minimize LCC of a personal computer.

Genetic Algorithm is a subclass of evolutionary algorithms, where the elements of the search space $G$ are binary strings $\left(G=B^{*}\right)$ or arrays of other elementary types [16].

Genetic Algorithms are widely used in LCC and LCA applications for the following reasons:

- $\quad$ They are more efficient than others when number of variables increase;

- $\quad$ They don't have any problem with multi-objective optimization;

- They are suitable for applications dealing with component-based systems (a product could be seen as a chromosome and its components as genes).

\section{Proposed Model}

In this section a model to optimize product life-cycle costs and environmental impacts together is proposed. The model is based on NSGA-2 (Non dominated Sorting Genetic Algorithm). NSGA-2 is one of the most popular and tested Genetic Algorithms. It has three special characteristics: (i) fast non-dominated sorting approach, (ii) fast crowded distance estimation procedure and (iii) simple crowded comparison operator [5]. To perform NSGA-2 it was used GANetXL [14], an add-in for Microsoft Excel.

We have applied NSGA-2 to LCC / LCA optimization. We have defined an experimental scenario composed by a preliminary set of 3 simplified test cases (Table 3 ), plus the application to a real industrial case, concerning the design of a production line. The performances of NSGA-2 in the introductory test cases have been compared with other two optimization methods, based on linear programming, in order to check the goodness of the proposed model.

Table 1. Test parameters

\begin{tabular}{llll}
\hline & Data Input & Optimal Solutions & Constraint \\
\hline Test A & A & Unique & No \\
Test B & B & Pareto Front & No \\
Test C & B & Pareto Front & Yes (1) \\
\hline
\end{tabular}

In the introductory tests, the life-cycle of a generic product made of 10 subgroups is evaluated. Each subgroup has two alternatives. The data input is composed by 4 types of cost and of 3 kinds of environmental impact. The models must optimize two 
objectives: minimize the life-cycle-cost and minimize the product life-cycle environmental impact.

Test A was used to see if all the models reach the unique optimal solution, while Test $\mathrm{B}$ and Test $\mathrm{C}$ were used to see the behaviour of the 3 models with multiple optimal solutions. Test $\mathrm{B}$ has no constraints, while Test $\mathrm{C}$ has a constraint.

In Test A, all the models reached the optimal solution. In Test B and in Test $\mathrm{C}$, the behaviour of the models were different.

NSGA-2 returns a number of solutions greater than the others two. Also its solutions are not dominated and some of them are surely optimal (compared to WSM, Weighted Sum Model). Then, it is possible to say that NSGA-2 is better than linear programming-based models.

The NSGA-2 model was then applied to a real case, a fraction of an assembly line, designed and manufactured by an Italian company. This line assemblies a small car diesel engine. Five stations were considered: the first is for silicon coating, the second assemblies the base, in the third screws are filled in, the fourth fills screws and rotates the pallets, the last screws the under base. All of these stations can have automatic, semi-automatic or manual alternatives. Each station has 6 alternatives: 3 automatic, 2 semi-automatic and 1 manual. 8 costs and 2 environmental impacts were considered for being optimized. In this case, the algorithm chromosome represents the line and stations represent the genes. The model has two types of constraints: the availability of the fraction of the assembly line must be greater than 0.95; all the stations must have an alternative. Below we report the model written in analytical form.

$$
\begin{gathered}
\min \sum_{i=1}^{30}\left(\text { Cin } * x_{i}+\operatorname{Ce} * x_{i}+\text { Cric } * x_{i}+\text { Cop } * x_{i}+\text { Ccon } * x_{i}+\text { Cair } * x_{i}+\right. \\
\text { Cmo } \left.* x_{i}+\text { Cmorip } * x_{i}\right) \\
\min \sum_{i=1}^{30}\left(\text { EIst } * x_{i}+\text { EIel } * x_{i}\right)
\end{gathered}
$$

Subject to

$$
\begin{gathered}
\sum_{i=1}^{6} A_{i} x_{i} * \sum_{i=7}^{12} A_{i} x_{i} * \sum_{i=13}^{18} A_{i} x_{i} * \sum_{i=19}^{24} A_{i} x_{i} * \sum_{i=25}^{30} A_{i} x_{i} \geq 0.95 \\
\sum_{i=1}^{6} x_{i}=1 \\
\sum_{i=7}^{12} x_{i}=1 \\
\sum_{i=13}^{18} x_{i}=1 \\
\sum_{i=19}^{24} x_{i}=1 \\
\sum_{i=25}^{30} x_{i}=1 \\
x_{i} \in\{0,1\} \quad i=1,2, \ldots, 30
\end{gathered}
$$

where: $C i n$ is initial cost, $C e$ is energy cost, Cric is spare parts cost, Cop is labor cost, Ccon is consumable cost, Cair is air cost, Cmo is preventive maintenance cost, Cmorip is corrective maintenance cost, EIst is environmental impact of the station and EIel is environmental impact of electric energy. $A$ is availability and $x_{i}$ is binary variable. 
So the model must optimize costs and environmental impacts along the product's life cycle, or rather the model must find the best combination of station to optimize the two objective.

Two scenarios were studied: one where the line is installed in Eastern Europe and one where the line is installed in Western Europe. The differences are the labor and maintenance staff costs: in fact in Western Europe they are 3 or 4 times greater than to Eastern Europe.

Fig. 1 and 2 report the results in Eastern Europe and Western Europe scenario.

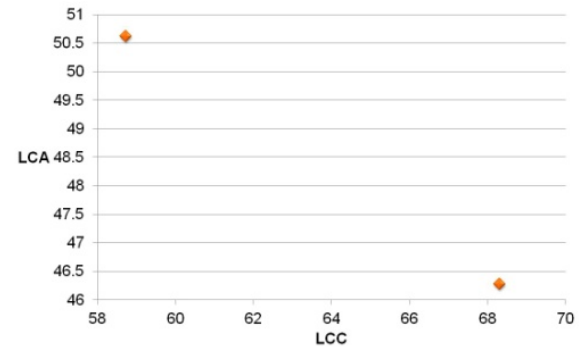

Fig. 1. Results (Eastern Europe Scenario)

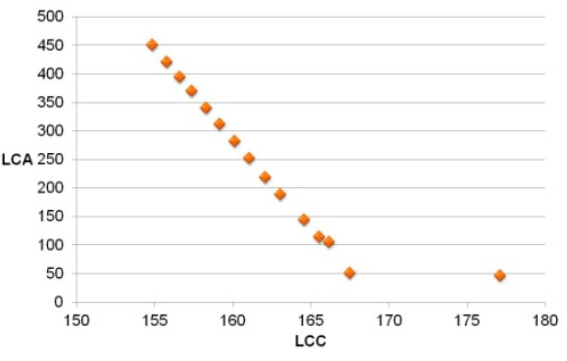

Fig. 2. Results (Western Europe Scenario)

In Eastern Europe Scenario the solution, that minimizes life-cycle cost, is composed of all manual stations, while in Western Europe the solution is composed of all automatic stations. This happens for differences in labor costs: in Eastern Europe, where labor costs are lower, there's convenience to install manual stations, instead in Western Europe it agrees automatic stations. To validate the obtained results, they were subjected to company. It has considered correct the solutions.

\section{Conclusion}

In this paper, a model is proposed to optimize product life-cycle costs and environmental impacts together. It was tested and compared to other two models. Then it was applied to a real case and the results have been validated. This presents some advantages, as (i) relevance for real case and (ii) comparison with other two models, and some criticism, as (i) performances are not evaluated and (ii) LCA not well investigated. The future developments can be (i) the inclusion of effectiveness / performance equations and (ii) the deepening of LCA.

\section{References}

1. Azapagic, A., Clift, R.: Life Cycle Assessment and Linear Programming -Environmental Optimisation of Product System. Computers Chemical Engineering 19, 229-234 (1995)

2. Azapagic, A., Clift, R.: Life cycle assessment and multiobjective optimisation. Journal of Cleaner Production 7, 135-143 (1998) 
3. Barringer, H.P.: A Life Cycle Cost Summary. In: ICOMS 2003 (2003)

4. Cattaneo, E.: L'Ottimizzazione della Progettazione tramite il Life-Cycle Cost. Thesis (2009)

5. Deb, K., Pratap, A., Agarwal, S., Meyarivan, T.: A fast and elitist multiobjective genetic algorithm: NSGA-II. Evolutionary Computation. IEEE Transactions 6, 182-197 (2002)

6. Dufo-Lopez, R., Bernal-Agustin, J.L., Yusta-Loyo, J.M., Dominguez-Navarro, J.A., Ramirez-Rosado, I.J., Lujano, J.: Aso: Multi-objective optimization minimizing cost and life cycle emissions of stand-alone PV-wind-diesel systems with batteries storage. Applied Energy 88, 4033-4041 (2011)

7. Frangopol, D.M., Liu, M.: Multiobjective Optimization for Risk-based Maintenance and Life-Cycle Cost of civil infrastructure systems. In: IFIP International Federation for Information Processing, vol. 199, pp. 123-137. Springer, Boston (2006)

8. Gitzel, R., Herbort, M.: Optimizing life cycle cost using genetic algorithms. Journal of Cost Management 22, 34-47 (2008)

9. Hinow, M., Mevissen, M.: Substation Maintenance Strategy Adaptation for Life-Cycle Cost Reduction Using Genetic Algorithm. IEEE Transactions on Power Delivery 26, 197-204 (2011)

10. Kaveh, A., Laknejadi, K., Alinejad, B.: Performance-based multi-objective optimization of large steel structures. Acta Mechanica 223, 355-369 (2011)

11. Kornelakis, A.: Multiobjective Particle Swarm Optimization for the optimal design of photovoltaic grid-connected systems. Solar Energy 84, 2022-2033 (2010)

12. Okasha, N.M., Frangopol, D.M.: Lifetime-oriented multi-objective optimization of structural maintenance considering system reliability, redundancy and life-cycle cost using GA. Structural Safety 31, 460-474 (2009)

13. Scientific Applications International Corporation: Life Cycle Assessment: Principles and Practice. Technical Report, 80 (2006)

14. Savić, D.A., Bicik, J., Morley, M.S.: A DSS Generator for Multiobjective Optimisation of Spreadsheet-Based Models. Environmental Modelling and Software 26, 551-561 (2011)

15. Wang, K., Dai, L., Myklebust, O.: Applying Particle Swarm Optimization (PSO) in Product Life Cycle Cost Optimization. IPROMS, 6 (2009)

16. Weise, T.: Global Optimization Algorithms - Theory and Application. Self Published, 820 (2009) 